pregnancy. Assessment of adrenal function should be preserved for newborns with clinical suspicion of adrenal dysfunction or with risk factors as prematurity or placenta bed pathology.

\section{PS-316 UMBILICAL BLOOD FLOW PATTERNS DIRECTLY AFTER BIRTH BEFORE DELAYED CORD CLAMPING}

${ }^{1}$ B Boere, ${ }^{2}$ AAW Roest, ${ }^{3} \mathrm{E}$ Wallace, ${ }^{4} \mathrm{ADJ}$ ten Harkel, ${ }^{5} \mathrm{M}$ Haak, ${ }^{6} \mathrm{CJ}$ Morley, ${ }^{3} \mathrm{SB}$ Hooper, ${ }^{7} \mathrm{AB}$ te Pas. ${ }^{\prime}$ Pediatrics, Leiden University Medical Center, Leiden, Netherlands; ${ }^{2}$ Pediatric Cardiology, Leiden University Medical Center, Leiden, Netherlands; ${ }^{3}$ The Ritchie Centre Monash Institute for Medical Research, Monash University, Melbourne, Australia; ${ }^{4}$ Pediatric Cardiology, Leiden University Medical Center, Leiden, Netherlands; ${ }^{5}$ Obstetrics and Gynaecology, Leiden University Medical Center, Leiden, Netherlands, ${ }^{6}$ Obstetrics and Gynaecology, University of Cambridge, Cambridge, UK; ${ }^{7}$ Pediatrics, Leiden University Medical Center, Leiden, Netherlands

\subsection{6/archdischild-2014-307384.614}

Background Delayed cord clamping (DCC) effects both cardiopulmonary transition and blood volume in neonates. Understanding the circulation through the umbilical vessels immediately after birth, with cord and placenta intact, is important.

Objective To describe the duration and patterns of blood flow through the umbilical vessels during DCC.

Methods Arterial and venous umbilical blood flow was measured during DCC using Doppler ultrasound in a prospective, observational, study of uncomplicated term vaginal deliveries. Immediately after birth, the probe was placed in the middle of the umbilical cord and the pattern and duration of flow in the vein and arteries evaluated until cord clamping.

Results Thirty infants were studied. Venous: In 10\% there was no flow, in $57 \%$ flow stopped at a median (IQR) min:sec of $4: 34(3: 03-7: 31)$ after birth before cord was clamped, and in $33 \%$ flow continued until cord clamping at 5:13 (2:56-9:15). Venous flow was initially intermittent $(100 \%$ increase during large breaths, stopped/reversed during crying), but became continuous. Arterial: In $17 \%$ there was no flow, in $40 \%$, flow stopped at 4:22(2:29-7:17), while cord pulsations were still palpable. In $43 \%$ flow continued until cord was clamped at 5:16 (3:32-10:20). Arterial flow was pulsatile, unidirectional towards placenta or bidirectional to/from placenta. In $40 \%$ flow became almost continuous (non pulsatile) later after birth.

Conclusion During DDC venous and arterial umbilical flow occurs for longer than previously described. Net placental transfusion is probably the result of several factors of which breathing could play a major role. Umbilical flow is unrelated to cessation of pulsations.

\section{PS-317 QF-PCR AS A STAND-ALONE TEST FOR DIAGNOSIS OF ANEUPLOIDES IN PRE AND POSTNATAL SAMPLES: AN INDIAN REPORT}

S Agarwal, S Muthuswamy. Medical Genetics, Sanjay Gandhi Postgraduate Institute of Medical Sciences, Lucknow, India

\subsection{6/archdischild-2014-307384.615}

Down syndrome is the most common aneuploides seen in live born babies with the prevalence of 1 in 1000 followed by other trisomies. The burden of aneuploides can be reduced with new molecular cytogenetic technology which helps in early intervention and genetic counselling. As our centre is being a referral centre for all genetic disorders, we receive a high risk couples related to chromosomal abnormalities as having one affected child. Since we are providing QF-PCR as a stand-alone test for postnatal diagnosis, the same methodology was extrapolated for prenatal diagnosis.

Initially, 500 postnatal samples (Blood in EDTA vial) and 240 amniotic fluid samples were received for analysis of chromosomal aneuploidies from various part of the country. The DNA was extracted with QIAamp DNA mini kit by Qiagen. QF-PCR was carried out with the following markers D21S1411, D21S11, D21S1435, D21S1412, AMEL, SRY, D18S535, D18S391, D13S258, D13S634 and XHPRT, X22 whose heterozygosity have been studied.

We observed 100\% concordance with the clinical diagnosis as well as cytogenetic analysis of postnatal samples. With these results we went for prenatal services were chromosomal studies are very common for suspected Down syndrome pregnancies. Out of 240 pregnancies studied, 2\% (5) was of Trisomy 21 and a single case of Trisomy 18 was identified, these results were also reconfirmed with karyotyping results.

Results of present investigation reassure QF-PCR as standalone test for high risk pregnancies. This is the first study from India that tested the in-house developed multiplex QF-PCR for post and prenatal diagnosis.

\section{PS-318 ARE ANTENATAL CORTICOSTEROIDS LESS EFFECTIVE IN TWINS THAN IN SINGLETONS? A COHORT STUDY IN THE ITALIAN NEONATAL NETWORK}

${ }^{1} \mathrm{~L}$ Gagliardi, ${ }^{2} \mathrm{R}$ Lucchini, ${ }^{2} \mathrm{~S}$ Giampietro, ${ }^{3} \mathrm{R}$ Bellù, ${ }^{3} \mathrm{R}$ Zanini. ${ }^{1}$ Woman and Child Health, Ospedale Versilia, Viareggio, Italy; ${ }^{2}$ Department of Pediatrics and Child Neuropsychiatry, Sapienza University, Roma, Italy; ${ }^{3}$ Neonatal Intensive Care Unit, Alessandro Manzoni Hospital, Lecco, Italy

\subsection{6/archdischild-2014-307384.616}

Background Although antenatal corticosteroids (ACS) represent the paradigm of "evidence-based medicine", their efficacy in case of twin pregnancy is not established, and available results are conflicting.

This study aimed at evaluating the association between ACS and neonatal outcomes in twins, comparing the results with those in singletons.

Methods A cohort of neonates 24-29 weeks gestational age (GA) without congenital anomalies, born in 2005-2012 and assisted in 90 hospitals adhering to the Italian Neonatal Network, was analysed.

Outcomes were: death, grade 3-4 intraventricular haemorrhage (IVH), cystic periventricular leukomalacia (PVL). Logistic regression models, adjusting for GA, sex and birthweight and clustering for hospitals, were used. Results were also checked adjusting for a propensity score of receiving ACS.

Results We studied 13029 infants (mean GA 27.1 weeks; mean birthweight $964 \mathrm{~g}$ ); $81.4 \%$ were treated with ACS (any dose); $29 \%$ were twins. Twins were treated more often than singletons (84.4 vs. $80.2 \%, \mathrm{p}<0.001)$.

Among twins, ACS were associated with a reduction of inhospital death (adjusted Odds ratio, aOR $=0.75 ; 95 \% \mathrm{CI}$ : $0.58-0.98)$ and IVH (aOR $=0.52 ; 95 \%$ CI: $0.40-0.68)$ but not PVL (aOR $=0.92$; CI: 0.62-1.36).

The effect of ACS was always smaller in twins than in singletons $(72 \%$ lower for death, $23 \%$ for IVH, and $15 \%$ for PVL).

Analyses using the propensity score approach yielded similar results.

Conclusions This large cohort allowed us to clarify that ACS prophylaxis is efficacious also in twins but the magnitude of the 